\title{
Development of an expert system framework for lifestyle improvement
}

\author{
Tamás Lőrincz, Benedek Szakonyi, Ágnes Lipovits, István Vassányi \\ Faculty of Information Technology, University of Pannonia \\ Veszprém, Hungary \\ tamas.lorincz@virt.uni-pannon.hu, benedek.szakonyi@virt.uni-pannon.hu, \\ lipovitsa@almos.uni-pannon.hu, vassanyi@almos.vein.hu
}

\begin{abstract}
In modern societies new, lifestyle related chronic diseases are appearing, affecting more and more people. Besides decreasing the quality of life for these patients, their treatments require increasing financial and social support from governments (and in many cases, even from the patients themselves). Apart from socio-economic concerns, another serious problem is the increasing shortage of experts (e.g. doctors, dietitians, ergonomists) that could help people, as the need for them is growing faster than their numbers. In this paper, a framework for creating expert systems capable of containing and properly using the knowledge of such experts, for providing help to users in acquiring and maintaining a healthy lifestyle, is presented. By selecting two different areas, diet and physical oriented lifestyle management and workplace related ergonomics, the effectiveness of such systems is tested.
\end{abstract}

Keywords-CLIPS; expert system; kinect; lifestyle assessment; telemedicine; work ergonomics;

\section{INTRODUCTION}

It is broadly accepted that good health pays a great role in general welfare, and that promoting and helping to achieve a healthy lifestyle is important, especially since more and more "modern civilization related problems" appear.

Non-communicable diseases (NCD) which, among others, include diabetes, cardiovascular and chronic respiratory conditions, are the leading causes of disability and death, moreover, they also represent a significant economic burden for both society and the patients. However, through proper interventions on factors like physical and mental activity, diet, unhealthy habits (e.g. smoking, excessive alcohol consumption) etc., prevention can reduce the risk of developing NCD [1].

Traditionally, such interventions are personalized, suggested and monitored by experts (e.g. doctors, dietitians, trainers, coaches etc.), who are in direct contact with the patients. However, the need for such "treatments" is considered to increase faster than the number of the available experts, creating a shortage of manpower (especially in medical personnel) [2]. On the other hand, such services are not yet provided globally, and where already accessible, these treatments usually require additional financial expenses, which may not be covered by insurances (if provided at all), so it is another factor that negative affects health equity.

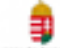

SUPPORTED BY THE ÚNKP-17-2 NEW NATIONAL EXCELLENCE PROGRAM OF THE MINISTRY OF HUMAN CAPACITIES

We acknowledge the financial support of Széchenyi 2020 under the EFOP-3.6.1-16-2016-00015
The field of telemedicine aims to overcome these barriers, to improve access to medical services by utilizing the inventions of information technology and telecommunication. By creating software products that incorporate the knowledge of human experts - i.e. by creating expert systems - it is possible to offer the aforementioned services to larger parts of societies: what makes this achievable, for example, is that the number of personal computers and mobile devices (smartphones, tablets etc.) has raised rapidly in the recent years and is expected to increase even more [3]. As these devices are becoming an almost inevitable part of everyday life (especially for younger generations), it seems almost trivial, that using them as the tools of treatment and prevention is an opportunity not to be missed out. By developing user friendly lifestyle assessing applications, it may prove to be possible to remotely help patients in establishing a more health-centered way of living, reducing both the number of chronic diseases and the economic costs related to their treatment.

With this in mind, an expert system framework was developed, that could serve as a basis for computerizing different factors of improving the quality of life. To test its capabilities, two distinct areas were selected: diet and physical oriented lifestyle management, and workspace related ergonomics.

In section II, after introducing the general concept of the developed framework (A), its technological properties are presented (B), followed by the two specific implementations, lifestyle management (C) and work ergonomics (D).

\section{THE FRAMEWORK}

\section{A. Expert System}

Transferring human knowledge to a computer is a difficult task, which can be solved by several different approaches. In case of representing a task-specific knowledge, artificial intelligence provides a useful tool, rule-based system. It contains predefined IF-THEN rule statements (called rule base) symbolizing the expert's (e.g. a dietitian) knowledge. Instead of using algorithmic solutions (procedural programming), the lifestyle of the user, and the work ergonomics can be assessed properly with rules related to the field.

After a thorough analysis, CLIPS was chosen, because of its advantages such as portability ( $\mathrm{C}$ code); low resource 
requirements; public domain software. There are various examples of critical projects where CLIPS proved its reliability (e.g. NASA used it in space shuttle missions [4] and security purposes [5]).

Another important component of the knowledge base is the fact. The inference engine can apply rules on the inserted facts to deduce new facts, even with including explanation. In most cases, the default behavior of CLIPS is enough to (partially) substitute a human expert. The previous statement does not hold when the problem is dynamic, considering time. It means, that the decision (consequences of the applied rules on facts) made by the system, is a function of time. This goal can be achieved by tracking the time of insertion of the facts, and formulating the rules with respect to that. It is very important to handle time passing: some facts might lose their actuality, and as a result, they would have to be updated or removed, while the need to insert some new facts could also arise, based on the time interval that has passed. For example, a fact like "the user was out running today" on the next day should be "the user was out running yesterday". With the appropriate time logging the identification of recurring events could be easily achieved, thus the discovery of behavior patterns could be possible. There are two possible solutions to deal with the "time problem". One of them allows us to have a very precise time information, assigning a timestamp to every fact. The disadvantage of this approach is that it is very far from human-like thinking. The way experts formulate rules tend to use the present time as reference.

To overcome this problem a relative timestamp (age) is assigned to each fact. It is crucial to handle more dimensions of time (e.g. minute, hour, day, week), since the rules defined by the experts are more likely to be formulated using different measure of time. The reader can find examples of these rules in (C). The expert system has aging rules, which are responsible for aging the inserted facts in a proper manner. These rules will activate (fire), when a special fact (which indicates the interval is passed) is inserted into the system. Generating these rules and adding the time dimensions to each fact is automatic as described in (B).

\section{B. Software}

The framework is written in $\mathrm{C}++$ programming language for the sake of maintaining the portability of CLIPS. It uses a database as a resource to load the rule sets, templates (structure of a fact), and to store the previously generated (deducted) facts, which are important when the environment needs to be restored. The database model of the expert system framework is shown on Fig. 1.

By organizing the rules into sets, it is possible to define different rules for different groups of people. Every time a fact is inserted into the framework, it is stored in the database to provide the ability of restoring a previous state. When a rule has a consequence and generates a deducted fact, this fact is stored in the fact table. The same rule might generate a consequence outside the framework (e.g. displaying a message on the user interface), these consequences are called events. If an event is generated, the framework assigns the rule to the event, queries for the proper message to display in the rule_gui table, and then sends it to the user interface. There are two tables, which are only necessary when the framework is being initialized. The facts contained by the deffact table are always inserted when the expert system starts. The templates are defining the structure of the facts, these are stored in the template table. Examples for these are shown in (C).

The developed framework is capable of handling two different configurations of time dimensions, which can be seen in Table I.

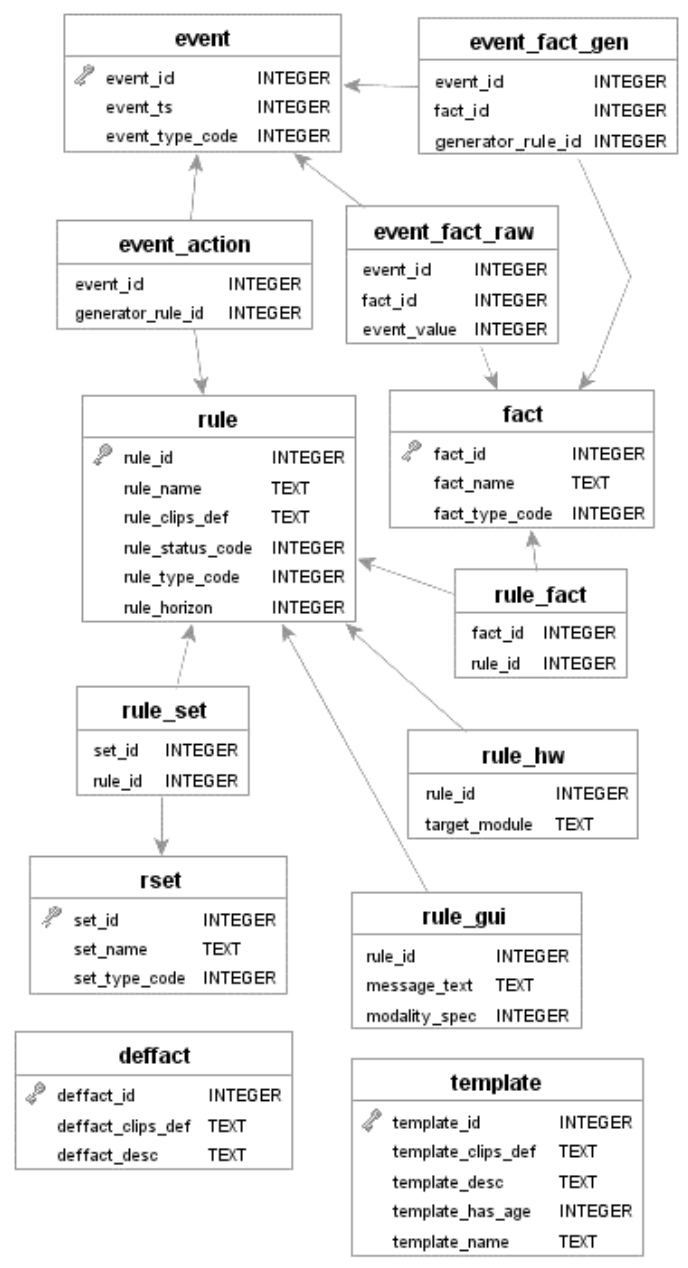

Fig. 1. Database model of the expert system framework.

The first one is suitable for applications, where the smallest time interval can be measured in one minute. In this case the facts in the framework has 2 time units: minute and hour. The second configuration is used, when the smallest relevant time interval is one hour, it means the framework can handle 3 dimensions of time: hour, day and week.

The framework provides a simple interface for the applications by hiding the complex mechanisms responsible for maintaining CLIPS, aging algorithms, and the database. Through this interface it is possible to change rule sets, assign the function which handles the consequences (e.g. notifying the user), and insert facts. There is a special function defined in this interface, that is responsible for signaling the expert system of time passing. When this function is called, the expert system will start aging the facts by the smallest time unit (minute or hour) previously set in the configuration (Table I.). 
TABLE I. TIME CONFIGURATIONS

\begin{tabular}{|c|c|c|}
\hline Configuration & Time units & Used in \\
\hline MINUTE_HOUR & minute, hour & $\begin{array}{c}\text { Work } \\
\text { Ergonomics }\end{array}$ \\
\hline HOUR_DAY_WEEK & $\begin{array}{c}\text { hour, day, } \\
\text { week }\end{array}$ & $\begin{array}{c}\text { Lifestyle } \\
\text { Management }\end{array}$ \\
\hline
\end{tabular}

\section{Lifestyle Management}

Of the treatments for the newly appearing diseases - which affect significant parts of the population - in today's industrialized societies, many are closely related to changing the patients' lifestyles. Living an unruly, careless, and thus unhealthy lifestyle has a great impact on developing these unwanted conditions. The Lavinia Lifestyle Application [6] helps users to log their daily nutrition and medicine intake, their physical activity. By assessing the data recorded this way, Lavinia aims to provide personalized proposals for the users, in order to help them achieve a more appropriate way of living. To make these recommendations available and as efficient as possible, the knowledge of dietary experts was needed. To achieve this by using the expert system mentioned in subsections (A-B), formulation of the "rules" used by dietitians was required.

Such a rule, for example, is the following: "If for the last week, a user exercised every day, then they should be complimented". To formalize such a rule, the fact that the user did some kind of an exercise must be logged and forwarded to the expert system (where aging of this rule is handled by the framework). It is easy to see, that by the nature of the framework it simple to define a rule that checks if there were exercises in the last 7 days. If the system can find 7 days where the age of the facts are respectively $0,1, \ldots, 6$ days, then the compliment happens. After this, a new (deducted) fact stating that the user received the compliment (or, in other cases, the warning) should be generated in order to avoid bothering the user by sending the same message over and over again, every time system evaluates the facts. Since the deducted fact also have an age, a rule must be defined, which removes this fact after one day. By removing the previous fact, the expert system is now allowed again to compliment the user if the conditions hold.

Another implemented rule is the following: "If the user reached his or her recommended daily fiber intake, a compliment must be sent." Two different messages belong to the compliment based on the user's aim regarding his or her weight (losing, gaining or maintaining). To formalize this rule, it was necessary to generate a (deducted) fact at every midnight. Another rule was needed, which takes care of setting the value of the daily fiber intake to zero, when it is midnight. A rule is defined, which fires when the user logs a meal intake, and in this case it adds the amount of fiber contained by the meal to the daily fiber intake. With the previous set of rules the expert system is aware of the current daily fiber intake of the user. Complimenting the user can be done with two more rules. Both of the rules have the condition which says the daily recommended fiber intake is reached. The first rule has one more special condition, which indicates that gaining weight is one of the user's goal. The other one applies for those, who would like to maintain or lose weight. The reader can find an example of the first rule on Fig. 2. (defrule

daily_fiber_intake_reached_compliment_healthy_maintain_or_gain_ weight

(not (user_illness)) (or (user info (weight goal maintain))

(user_info (weight_goal gain))

(not (fiber_intake_reached_feedback_given))

(fiber_intake_reached)

(clips_event_happened 2) (assert (fiber_intake_reached_feedback_given))

Fig. 2. Example of a CLIPS rule

In order to test the implemented rules test cases were defined. With respect to the physical activity rule (exercise every day through a week) three different cases can be distinguished:

- The physical activity of the user is irregular, so in the behavior pattern exercising every day in week does not appear. In this case, the expert system should not send a compliment.

- The user's log contains physical activities through exactly 7 days in a row (but not on the $8^{\text {th }}$ day). It means the expert system should send a compliment right after the last activity.

- The user logs physical activities more than 7 days in a row, which means the expert system should compliment him or her after every activity starting from the $7^{\text {th }}$ day.

More test cases were defined for the other implemented rules, and the tests resulted properly. It is important to note that the previously defined tests should result correctly in case of implementing a new function. By running these tests after every improvement of the rule set the consistency of the rules can be granted.

For the data (e.g. meal intakes, physical activities, user information) coming from the Lavinia application a Protocol Buffers (Protobuf) interface was defined. By using this technology, it is possible to send structured information. The application only transfers data to the expert system which is relevant for assessing the lifestyle. The construction of these messages is shown on Fig. 3.

\begin{tabular}{|c|c|}
\hline Fact & \\
\hline \multicolumn{2}{|c|}{$\begin{array}{l}\text { enum FactType } \\
\text { \{ } \text { PHYSICAL_ACTIVITY = } 0 ; \text { MEAL_INTAKE = } 1 ; \\
\text { USER_INFO }=2 ; \quad \operatorname{RDA}=3 ;\} \\
\text { FactType fact_type }=1 ; \\
\text { bytes data }=2 ;\end{array}$} \\
\hline MealIntake & PhysicalActivity \\
\hline $\begin{array}{l}\text { double fiber=1; } \\
\text { double fat=2; } \\
\text { double kcal=3; }\end{array}$ & $\begin{array}{l}\text { double } k c a l=1 ; \\
\text { double duration=2; }\end{array}$ \\
\hline RDA & UserInfo \\
\hline $\begin{array}{l}\text { double fiber_min }=1 ; \\
\text { double fiber_opt }=2 ; \\
\text { double fiber_max }=3 ; \\
\qquad \ldots\end{array}$ & $\begin{array}{l}\text { enum WeightGoal } \\
\{\text { MAINTAIN = } 0 ; \text { LOSE = } 1 ; \\
\text { GAIN = } 2 ;\} \\
\text { int32 weight_kg = } 1 ; \\
\text { WeightGoal weight_goal = } 2 \text {; }\end{array}$ \\
\hline
\end{tabular}

Fig. 3. Construction of messages from Lavinia to the expert system 


\section{Work Ergonomics}

For developed countries, already at the beginning of the 2000s, computer based working was dominant [7]. The number of people spending their working hours in front of computers (mainly in office environment) increased since then, and it can be expected to keep increasing in the years to come $[8,9]$. Thus, when trying to assist in having a proper lifestyle, it should also be investigated if decent work conditions are provided or not. As computer usage causes spending most of the work time being seated [10], one of the essential aspects in health preservation is posture allocation. What kind of postures does a person take on during the (usually long) work hours? Are they more likely to stoop in front of the screen all day, or do they stay in a good and ergonomically proper posture? How is it possible to constantly and efficiently monitor and "measure" the user's behavior (without disturbing the person repeatedly, which would mean decreasing work performance)? As in other problems and aspects of everyday life the developments and gadgets of information technology seem to have an ever increasing role in providing assistance, it would be thoughtful to investigate how this tendency can be utilized in solving this workplace issue.

The problem can be separated into two main tasks:

- Computerizing the required knowledge (which in this case is the expertise of ergonomists) in order to eliminate the need of the constant presence of an expert.

- Finding a way to obtain the user input (how the person sits, stands etc.) without needing any active cooperation from the user

As already discussed, by applying an expert system it is possible to complete the first task efficiently. As most office works require a computer, deploying such an expert system software is possible (of course, by keeping in mind that the computer performance should not be decreased noticeably).

For the second task, using some kind of camera equipment would seem trivial, but in order to properly detect the user's position and posture, acquiring 3 dimensional information is needed. For this, either 2 or more 2D cameras would be needed, or a sensor that is capable of recording 3D data. While the former would be possible, as it requires more effort to handle (correctly positioning the cameras, frame synchronization etc.), it is preferable to have a single device that is capable of providing the desired input. Such a device is the Microsoft Kinect V2 sensor, which can provide both color, infra-red and depth data without any need of markers placed, moreover, its built-in key features include body recognition and tracking (up to 6 people, 25 skeletal joints each), that could serve as the main data source for posture identification. To test whether the Kinect is applicable for ergonomic evaluation, a test software was created that can measure, display and record the user's behavior, and at the time of writing this paper, it was being evaluated by ergonomist experts. Based on early results, the Kinect sensor seems to prove utilizable, yet preprocessing of "raw Kinect data" is found to be necessary, as for some cases the joint position estimations resulted in unnatural body poses or false body detections. By changing where and how the device was positioned and oriented, it was found that there are some more preferable and less desirable settings for achieving accurate readings, what corresponds to the findings of [11].

In parallel to the Kinect testing, a basic evaluating software (using the framework developed) was created, to evaluate whether the system can react to some basic situations - like spending "too much" time in one sitting - correctly or not. As expected, by using proper rulesets and inputs, the expert system could produce the appropriate outputs.

In early stages of both test procedures, the concept of having the system run on an additional computer, not on the one the user operates, have arisen. Partly because of security reasons (to make it harder for unauthorized accesses to get the data used), partly to "spare" the user's machine from the computational needs of Kinect's data procession, and partly to provide error resistant environment (e.g. defense against power outage caused data loss).

As a result, the idea of an "intelligent workstation" was born, where the optimal and fix positioning of the Kinect could be possible, along with separating the whole system from the "outside". Some other feature proposals, that could be found beneficial, were created, such as motorized desk height adjustment, which could allow both sitting and standing modes (in order to decrease sedentariness), and customizability for matching the physical characteristics of the user. An additional desk lamp with self-adjusting lighting settings, in order to adapt to the changing illuminance as time passes, or to provide mood improving tints, or to serve as an indicator of engagement (e.g. do not disturb) could also be useful.

\section{DISCUSSION}

A framework capable of representing the basic human knowledge of dietitian and ergonomist experts was developed, which, based on early test result, shows promise. Yet, further study on how more complex assessment of user behavior can be achieved, and what the corresponding system actions should be, is necessary.

Using the developed framework for assessing lifestyle, therefore helping the user to maintain a healthy life is promising. The rules given by dietitian experts are easy to formalize in the framework, however more rules need to be implemented for correctly evaluate the data coming from the Lavinia application. Sending positive feedbacks to the users about their advantageous decisions related to their lifestyle also helps to keep them engaged of using the software.

As mentioned, using Kinect as the main input device might be beneficial for posture detection. Moreover, it could allow biometrics based login for the workstation (e.g. face features in Windows Hello). However, this ability to capture such data raises serious questions on privacy issues. Mainly, because that the fact of being monitored may already be unpleasant for some users, even if done anonymously and without recoding any actual personal data (like face features). This means that, apart from legal reasons, users of such workstations should be granted the possibility of disabling such functions. And for those who choose to use them, it should be made clear that such sensitive data is kept safe (and, of course, besides the sake of building trust, such security measures should be implemented for real). 
The concept of an intelligent workstation embedding the capability of assessing user behavior is interesting, and may serve as further research material.

\section{REFERENCES}

[1] Franco Sassi, Jeremy Hurst, The Prevention of Lifestyle Related Chronic Disease: An Economic Framework. OECD Health Working Papers (2008), p. 32

[2] World Health Organization, Global Health Workforce Alliance. A Universal Truth: No Health Without a Workforce. Third Global Forum on Human Resources for Health Report, November 2013. ISBN 978924 1506762.

[3] Cisco Systems Inc. Cisco Visual Networking Index: Forecast and Methodology, 2016-2021. September 2017. Document ID: 1465272001663118

[4] John F. Muratore, Troy A. Heindel, Terri B. Murphy, Arthur N Rasmussen, Robert Z. McFarland, Applications of Artificial Intelligence to Space Shuttle Mission Control, Innovative Applications of Artificial Intelligence proceedings, ISBN 978-0-262-69137-6
[5] David Silberberg, Robert Thomas, The NASA Personnel Security Processing Expert System, Innovative Applications of Artificial Intelligence proceedings, ISBN 978-0-262-51091-2

[6] I. Kósa, I. Vassányi, M. Nemes, K.H. Kálmánné, B Pintér, L. Kohut, A fast, android based dietary logging application to support the life style change of cardio-metabolic patients. Med-e-Tel Conference, 9-11 April 2014, Luxembourg. In Malina Jordanova, Frank Lievens (eds.) Global Telemedicine and eHealth Updates: Knowledge Resources, Vol. 7 (2014), ISSN 1998-5509, pp. 553-556.

[7] Smith MJ, Conway FT, Karsh BT, Occupational stress in human computer interaction. Ind Health 1999;37:157-173.

[8] National Research Council. (2008). Research on future skill demands: A workshop summary.Washington, DC: The National Academies Press

[9] Autor, D. H., Levy, F., \& Murnane, R. J, (2003). The skill content of recent technological change: An empirical exploration. Quarterly Journal of Economics, 118, 1279-1333.

[10] Hill JO, Peters JC, Environmental contributions to the obesity epidemic. Science 1998;280:1371-1374

[11] Wiedemann, Lukas \& Planinc, Rainer \& Kampel, Martin, (2014). Ergonomic-Monitoring of Office Workplaces Using Kinect. . 10.1007/978-3-319-13105-4_40. 